\title{
AN INVERSE SCATTERING PROBLEM FOR ACOUSTIC WAVES IN A SPHERICALLY STRATIFIED MEDIUM*
}

\author{
by DAVID COLTON \\ (Received 9th September 1975)
}

\section{Introduction}

The inverse problem we will consider in this paper has its origins in the following problem connected with the scattering of acoustic waves in a nonhomogeneous medium. Let an incoming plane acoustic wave of frequency $\omega$ moving in the direction of the $z$ axis be scattered off a "soft" sphere $\Omega$ of radius one which is surrounded by a pocket of rarefied or condensed air in which the local speed of sound is given by $c(r)$ where $r=|x|$ for $x \in R^{3}$. Let $u_{s}(x) e^{i \omega t}$ be the velocity potential of the scattered wave and let $r, \theta, \phi$ be spherical coordinates in $R^{3}$. Then from a knowledge of the far field pattern $f(\theta, \phi ; \lambda)$ for $\lambda=\omega / c_{0}$ contained in some finite interval $0<\lambda_{0} \leqslant \lambda \leqslant \lambda_{I}$, we would like to determine the unknown function $c(r)$. Under the assumptions that $\nabla c(r)$ is small in magnitude compared with $\lambda c(r)$ and $c(r)=c_{0}=$ constant for $r \geqslant a>1$, we can formulate this problem mathematically as follows (c.f. (1)):

Let $B(r)=\left(c_{0} / c(r)\right)^{2}-1$ and set $u_{s}(x)=v(x)+u(x)$ where $u(x)$ satisfies

$$
\begin{gathered}
\Delta_{3} u+\lambda^{2}(1+B(r)) u=0 \text { in } R^{3} \backslash \Omega \\
u(x)=-\left(e^{i \lambda z}+v(x)\right) \text { on } \partial \Omega \\
\lim _{r \rightarrow \infty} r(\partial u / \partial r-i \lambda u)=0
\end{gathered}
$$

and $v(x)$ is such that $e^{i \lambda z}+v(x)$ is a solution of (1.1) in $R^{3} \backslash \Omega$ where $v(x)=0$ for $r \geqslant a$. Then given

$$
f(\theta, \phi ; \lambda)=\lim _{r \rightarrow \infty} r e^{-i \lambda r} u(x)
$$

we want to determine the function $B(r)$. The approach we will use in this paper is to use the theory of integral operators for partial differential equations in unbounded domains as recently initiated by Colton and Wendland in (1) to reduce the inverse scattering problem described above to a generalised moment problem for the unknown function $B(r)$. In this

*This research was supported in part by AFOSR Grant 74-2592. 
sense our work has some relation to the work of Rorres, who solved the inverse scattering problem for small frequencies in the absence of an obstacle by reducing the inverse problem to a moment problem over a finite interval (5).

\section{Integral Operators and the Inverse Scattering Problem}

Assume that $B(r)$ is continuously differentiable and vanishes for $r \geqslant a$, and let $h(r, \theta, \phi)$ be a solution of

$$
\Delta_{3} h+\lambda^{2} h=0
$$

in $R^{3} \backslash \Omega$. Then in (1) it was shown that every solution of (1.1) in $R^{3} \mid \Omega$ can be represented in the form

$$
\begin{aligned}
u(r, \theta, \phi) & =K[h] \\
& =h(r, \theta, \phi)+\int_{r}^{\infty} K(r, s ; \lambda) h(s, \theta, \phi) d s
\end{aligned}
$$

where $h(r, \theta, \phi)$ is a solution of $(2.1)$ in $R^{3} \backslash \Omega$, and, for $1 \leqslant r \leqslant s<\infty$, $K(r, s ; \lambda)$ can be represented in the form

with

$$
K(r, s ; \lambda)=(r s)^{-\frac{1}{2}} \sum_{j=0}^{\infty} \lambda^{2 j+2} N_{j}(\log r, \log s)
$$

$$
N_{0}(\log r, \log s)=-\frac{1}{2} \int_{\frac{1}{2} \log r s}^{\log a} e^{2 \tau} B\left(e^{\tau}\right) d \tau
$$

and the functions $N_{j}(\log r, \log s), j=0,1, \ldots$, being determined recursively. Each $N_{i}(\log r, \log s)$ is independent of $\lambda$, vanishes identically for $r s \geqslant a^{2}$ (since $B(r)=0$ for $r \geqslant a$ ), and satisfies a bound of the form

$$
\max _{1 \leqslant r \leqslant s<\infty}\left|N_{j}(\log r, \log s)\right| \leqslant C /(2 j+1) !
$$

where $C$ is a constant which is independent of $j$ and depends only on the maximum of $|B(r)|$ in the interval $1 \leqslant r \leqslant a$. In particular (2.5) implies that the series (2.3) is uniformly convergent for $1 \leqslant r \leqslant s<\infty$ and is an entire function of $\lambda$. Since $e^{i \lambda z}$ is a solution of (2.1), the above considerations imply that a suitable choice for $v(x)$ is given by

$$
v(x)=v(r, \theta)=\int_{r}^{\infty} K(r, s ; \lambda) e^{i \lambda s \cos \theta} d s .
$$

Now let $J_{n+\frac{1}{2}}(\lambda r)$ and $H_{n+\frac{1}{2}}^{(1)}(\lambda r)$ denote respectively a Bessel function and Hankel function of the first kind, and define $j_{n+\frac{1}{2}}(r)$ and $h_{n+\frac{1}{2}}(r)$ by 


$$
\begin{aligned}
j_{n+\frac{1}{2}}(r) & =K\left[(\lambda r)^{-\frac{1}{2}} J_{n+\frac{1}{2}}(\lambda r)\right] \\
h_{n+\frac{1}{2}}(r) & =K\left[(\lambda r)^{-\frac{1}{2}} H_{n+\frac{1}{2}}^{(1)}(\lambda r)\right] .
\end{aligned}
$$

Then from the representation (2, p. 64)

$$
e^{i \lambda z}=\sqrt{\frac{\pi}{2 \lambda r}} \sum_{h=0}^{\infty}(2 n+1) i^{n} J_{n+\frac{1}{2}}(\lambda r) P_{n}(\cos \theta)
$$

where $P_{n}(\cos \theta)$ denotes Legendre's polynomial, it is easily verified that the solution of (1.1)-(1.3) is given by

$$
u(x)=u(r, \theta)=-\sqrt{\frac{\pi}{2}} \sum_{n=0}^{\infty} \frac{(2 n+1) i^{n} j_{n+\frac{1}{2}}(1)}{h_{n+\frac{1}{2}}(1)} h_{n+\frac{1}{2}}(r) P_{n}(\cos \theta) .
$$

Note that from the uniqueness of the solution to (1.1)-(1.3) (1) we can conclude that $h_{n+\frac{1}{2}}(1) \neq 0$, and the convergence of the series (2.9) for $1 \leqslant r<\infty, 0 \leqslant \theta \leqslant \pi$ follows from (2.7) and well known estimates for Bessel functions and Legendre polynomials for large values of $n$ (c.f. (2), p. 22-23 and p. 205). From the fact that

$$
h_{n+\frac{1}{2}}(r)=(\lambda r)^{-\frac{1}{2}} H_{n+\frac{1}{2}}^{(1)}(\lambda r) ; \quad r \geqslant a
$$

and the asymptotic estimate (2, p. 85)

$$
H_{n+\frac{1}{2}}^{(1)}(\lambda r)=(-i)^{n+1} \sqrt{\frac{2}{\pi \lambda r}} e^{i \lambda r}\left[1+O\left(\frac{1}{\lambda r}\right)\right]
$$

we can conclude (c.f. (3)) that the far field pattern $f(\theta, \phi ; \lambda)=f(\theta ; \lambda)$ is given by

$$
f(\theta ; \lambda)=\sum_{n=0}^{\infty} \frac{i(2 n+1) j_{n+\frac{1}{2}}(1)}{\lambda h_{n+\frac{1}{2}}(1)} P_{n}(\cos \theta) .
$$

Recall once again that although the far field pattern $f(\theta ; \lambda)$ is assumed to be known, the functions $j_{n+\frac{1}{2}}(r)$ and $h_{n+\frac{1}{2}}(r)$ are unknown since $B(r)$ is as of yet unknown. However if we expand $f(\theta ; \lambda)$ in a Legendre series

$$
f(\theta ; \lambda)=\sum_{n=0}^{\infty} \tilde{a}_{n}(\lambda) P_{n}(\cos \theta),
$$

then from (2.12) and (2.13) we have

$$
\begin{aligned}
\frac{j_{n+\frac{1}{2}}(1)}{h_{n+\frac{1}{2}}(1)}= & a_{n}(\lambda) \\
= & \lambda^{2 n+1}\left(a_{n_{0}}+a_{n_{1}} \lambda^{2}+\cdots\right) \\
& +\lambda^{4 n+2}\left(c_{n_{0}}+c_{n_{1}} \lambda^{2}+\cdots\right)
\end{aligned}
$$


where

$$
a_{n}(\lambda)=\frac{\lambda \tilde{a}_{n}(\lambda)}{i(2 n+1)}
$$

are known (analytic) functions of $\lambda$. The fact that $a_{n}(\lambda)$ has a zero of order $2 n+1$ at the origin follows from (2.3), (2.7), and the series representations (c.f. (2), p. 4)

$$
\begin{aligned}
(\lambda r)^{-\frac{1}{2}} J_{n+\frac{1}{2}}(\lambda r)= & \sqrt{\frac{1}{2}} \sum_{m=0}^{\infty}(-1)^{m} \frac{(\lambda r / 2)^{2 m+n}}{m ! \Gamma\left(m+n+\frac{3}{2}\right)} \\
(\lambda r)^{-\frac{1}{2}} H_{n+\frac{1}{2}}^{(1)}(\lambda r)= & \sqrt{\frac{1}{2}} \sum_{m=0}^{\infty}(-1)^{m}\left[\frac{(\lambda r / 2)^{2 m+n}}{m ! \Gamma\left(m+n+\frac{3}{2}\right)}\right. \\
& \left.+i \frac{(-1)^{n+1}(\lambda r / 2)^{2 m-n-1}}{m ! \Gamma\left(m-n+\frac{1}{2}\right)}\right]
\end{aligned}
$$

Equating like powers of $\lambda$ in (2.14) we have for $n \geqslant 0$

$$
a_{n_{0}}=i \frac{(-1)^{n} \Gamma\left(-n+\frac{1}{2}\right)\left(\frac{1}{2}\right)^{2 n+1}}{\Gamma\left(n+\frac{3}{2}\right)}
$$

and, for $n>0$,

$$
\begin{aligned}
-\frac{\left(\frac{1}{2}\right)^{n+2}}{\Gamma\left(n+\frac{5}{2}\right)}+\int_{1}^{\infty} N_{0}(\log 1, \log s) \frac{\left(\frac{1}{2}\right)^{n} s^{n-\frac{1}{2}}}{\Gamma\left(n+\frac{3}{2}\right)} d s \\
=a_{n_{0}} i \frac{(-1)^{n}\left(\frac{1}{2}\right)^{-n+1}}{\Gamma\left(-n+\frac{3}{2}\right)}+a_{n_{1}} i \frac{(-1)^{n+1}\left(\frac{1}{2}\right)^{-n-1}}{\Gamma\left(-n+\frac{1}{2}\right)} \\
+a_{n_{0}} i \int_{1}^{\infty} N_{0}(\log 1, \log s) \frac{(-1)^{n+1}\left(\frac{1}{2}\right)^{-n-1} s^{-n-3 / 2}}{\Gamma\left(-n+\frac{1}{2}\right)} d s .
\end{aligned}
$$

The equation corresponding to $(2.18)$ for $n=0$ is exactly the same except that the term $c_{o_{0}} / \Gamma\left(\frac{3}{2}\right)$ is added to the right hand side. Note that the coefficient $a_{n_{0}}$ is independent of $B(r)$. From (2.4) we have

$$
\begin{aligned}
\int_{1}^{\infty} N_{0}(\log 1, \log s) s^{m} d s & =-\frac{1}{2} \int_{1}^{a^{2}} \int_{s^{1 / 2}}^{a} \xi B(\xi) s^{m} d \xi d s \\
& =-\frac{1}{2} \int_{1}^{a} \int_{1}^{\xi^{2}} \xi B(\xi) s^{m} d s d \xi \\
& =-\frac{1}{2(m+1)} \int_{1}^{a} \xi^{2 m+3} B(\xi) d \xi+\frac{1}{2(m+1)} \int_{1}^{a} \xi B(\xi) d \xi
\end{aligned}
$$

and hence using (2.17) and (2.19) we can rewrite (2.18) in the form 


$$
\mu_{n}=\int_{1}^{a} B(s)\left[s^{2 n+2}+s^{-2 n}-2 s\right] d s
$$

where for $n>0$

$$
\mu_{n}=-(2 n+1)\left[\frac{-(2 n+1)}{(2 n+3)(1-2 n)}+a_{n_{1}} i \frac{(-1)^{n+1}\left(\frac{1}{2}\right)^{-2 n-1} \Gamma\left(n+\frac{3}{2}\right)}{\Gamma\left(-n+\frac{1}{2}\right)}\right] .
$$

For $n=0, \mu_{0}$ is the same as defined by (2.21) except that the term $c_{o_{0}}$ is subtracted from the right hand side. The $\mu_{n}$ are known from the far field pattern, and hence the problem of determining the function $B(r)$ has been reduced to solving the generalised moment problem (2.20), (2.21) (note that if we assume that $B(r)$ is real valued, then from (2.17) and (2.18) we have that $a_{n_{1}}$ is purely imaginary, and hence $\mu_{n}$ is real for $\left.n=0,1, \ldots\right)$.

\section{The Generalised Moment Problem}

We will assume the existence of a continuously differentiable function $B(r)$ such that (2.20), (2.21) is valid, and address ourselves to the problem of the uniqueness of $B(r)$ and the approximation of $B(r)$ in the $L^{2}$ norm over the interval $[1, a]$. As will be clear from the analysis which follows, necessary and sufficient conditions on the sequence $\mu_{n}, n=0,1, \ldots$, for (2.20), (2.21) to determine a function $B(r) \in L^{2}[1, a]$ can be obtained from known results on the classical Hausdorff moment problem over the interval $\left[1 / a^{2}, a^{2}\right]$ (c.f. (4)). We however restrict ourselves solely to the problem of uniqueness and approximation since in the context of the present paper it is these problems which are of paramount interest. This is due to the fact that the sequence $\mu_{n}$ (or equivalently the sequence $a_{n_{1}}$ ) is obtained from physical measurement and it is assumed $a$ priori that the sequence $\mu_{n}$ is a (generalised) moment sequence for some function $B(r)$ to be determined.

The basic problems of uniqueness and approximation can be settled by appealing to the following theorem:

Theorem. The functions

$$
p_{n}(r)=r^{2 n+2}+r^{-2 n}-2 r,
$$

$n=0,1,2, \ldots$, are complete in $L^{2}[1, a]$.

Proof. Let $f(r)$ be a continuous function on the interval $[1, a]$. Since the space of continuous functions on $[1, a]$ is dense in $L^{2}[1, a]$, to prove the theorem it suffices to show that if

$$
\int_{1}^{a} f(s) p_{n}(s) d s=0
$$


for $n=0,1,2, \ldots$, then $f(r)=0$ for $r \in[1, a]$. For $r \in[1 / a, 1]$ define $f(r)$ by

$$
f(r)=r^{-4} f(1 / r) ; \quad r \in[1 / a, 1] \text {. }
$$

Then

$$
\int_{1}^{a} f(s) s^{-2 n} d s=\int_{1 / a}^{1} f(s) s^{2 n+2} d s
$$

and hence from (3.1)

$$
\begin{aligned}
0 & =\int_{1}^{a} f(s)\left[p_{n}(s)-p_{n+1}(s)\right] d s \\
& =\int_{1}^{a} f(s)\left[s^{2 n+2}+s^{-2 n}-s^{2 n+4}-s^{-2 n-2}\right] d s \\
& =\int_{1 / a}^{a} f(s)\left[s^{2 n+2}-s^{2 n+4}\right] d s \\
& =\frac{1}{2} \int_{1 / a^{2}}^{a^{2}} f\left(s^{\frac{1}{2}}\right)\left[s^{\frac{1}{2}}-s^{\frac{3}{2}}\right] s^{n} d s
\end{aligned}
$$

for $n=0,1,2 \ldots$ Since the set $\left\{r^{n}\right\}_{n=0}^{\infty}$ is complete in $L^{2}\left[1 / a^{2}, a^{2}\right]$, we have from (3.4) that

$$
f\left(r^{\frac{1}{2}}\right)\left(r^{\frac{1}{2}}-r^{\frac{3}{2}}\right)=0
$$

for $r \in\left[1 / a^{2}, a^{2}\right]$, and hence $f(r)=0$ for $r \in[1, a]$ and the theorem is proved.

The uniqueness of the function $B(r)$ follows immediately from the above theorem. Furthermore the function $B(r)$ can be approximated in $L^{2}[1, a]$ by orthonormalizing the set $\left\{p_{n}(r)\right\}_{n=0}^{\infty}$ over the interval $[1, a]$ to obtain the orthonormal set $\left\{\varphi_{n}(r)\right\}_{n=0}^{\infty}$ and then approximating $B(r)$ in $L^{2}[1, a]$ by the function

$$
B_{N}(r)=\sum_{n=0}^{N} b_{n} \varphi_{n}(r)
$$

where

$$
b_{n}=\int_{1}^{a} \varphi_{n}(s) B(s) d s .
$$

The coefficients $b_{n}$ can be found by using (2.20), (2.21), If it is assumed that $B(r) \in C^{1}[1, a]$, then it can be concluded that $B_{N}(r)$ approximates $B(r)$ pointwise almost everywhere on $[1, a](4$, p. 71$)$. Other approximation procedures are also of course available, for example by using (2.20), (2.21) to compute the Fourier cosine transform of $B(r)\left(r^{2}-r^{4}\right)$ over the interval $[1 / a, a]($ c.f. (5)). 


\section{REFERENCES}

(1) D. Colton and W. WEndland, Constructive methods for solving the exterior Neumann problem for the reduced wave equation in a spherically symmetric medium, Proc. Roy. Soc. Edinburgh Sect. A 75 (1976), 97-107.

(2) A. ERDÉlyI, et al., Higher Transcendental Functions, Vol. II (McGraw Hill, New York, 1953).

(3) P. Hartmann and C. Wilcox, On solutions of the Helmholtz equation in exterior domains, Math. Z. 75 (1961), 228-255.

(4) I. P. Natanson, Constructive Function Theory, Vol. II. (Frederick Ungar Publishing Co., New York, 1965).

(5) C. RORRES, Low energy scattering by an inhomogeneous medium and by a potential, Arch. Rational Mech. Anal. 39 (1970), 340-357.

DEPARTMENT OF MATHEMATICS

UNIVERSITY OF STRATHCLYDE

Glasgow, Scotland 